\title{
Laparoscopic Umbilical Hernia Repair Using a "Two-Port" Technique: A Single-Center Experience
}

\author{
Ghassan Almaimani ${ }^{\mathrm{a}}$,, Ahmad Oyais ${ }^{\mathrm{a}}$
}

\begin{abstract}
Background: Laparoscopic umbilical hernia repair has gained popularity over the last decade. As an alternative to the classical three- to four-port laparoscopic technique, the two-port technique is reported to have the advantages of being the least minimally invasive umbilical repair procedure described to date. The aim of this study was to determine the operative outcomes of patients treated using the twoport umbilical hernia repair technique.
\end{abstract}

Methods: This was a retrospective, single-center study. The case notes of 52 patients undergoing umbilical hernia repair between November 2015 and November 2016 via the two-port method were reviewed. Operative time and hospital stay were recorded. Data were analyzed using descriptive statistics.

Results: The operating time was $32 \mathrm{~min}$ on average. There was no significant procedural blood loss, iatrogenic injury, or subcutaneous emphysema. No patient required conversion to conventional methods. All patients were comfortable in the postoperative period and were routinely discharged on the second postoperative day. Forty-five of the 52 cases completed 3-month follow-up without any reported complications.

Conclusions: Excellent outcomes are possible using the two-port technique for umbilical hernia repair. However, proper patient selection and experience and proficiency in performing laparoscopic hernia repair are prerequisites for good outcomes.

Keywords: Umbilical hernia; Ventral hernia; Laparoscopy

\section{Introduction}

Despite refinements in laparoscopy equipment and techniques, there is still interest in reducing surgical trauma and improv-

Manuscript submitted June 13, 2017, accepted June 30, 2017

aDepartment of Surgery, HELIOS St-Elisabeth Klinik, Hunfeld, Germany

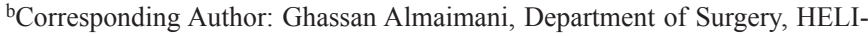
OS St-Elisabeth Klinik, Weinbergstr. 6a, 63571 Gelnhausen, Germany.

Email: dr.gaam@hotmail.com

doi: https://doi.org/10.14740/jcs329w ing esthetic quality by reducing the number and size of portal incisions. Umbilical hernia repair has undergone impressive developments in recent years, and laparoscopic mesh repair is now the treatment of choice for umbilical hernias due to its low recurrence rate, short hospital stay, and low complication rate $[1,2]$. Laparoscopic repair is classically carried out using three to four ports in the abdominal cavity $[3,4]$. However, the recently reported "two-port" technique has the added advantage of being the least minimally invasive procedure described to date $[1,2]$. Here we report our very favorable experiences of performing laparoscopic umbilical hernia repair using a twoport technique.

\section{Materials and Methods}

Fifty-two patients with umbilical hernias underwent two-port laparoscopic repair between November 2015 and November 2016. Informed consent was obtained from all patients. The same surgical team performed all operations. Operative time and hospital stay were recorded in each case. All patients were offered the two-port technique using Parietex mesh (Medtronic, Dublin, Ireland). No ethical approval was required since this was a retrospective review of service provision.

\section{Operative technique}

Patients were placed in the supine position with both arms tucked alongside. Monitors were placed on the right side of the bed. A second-generation cephalosporin was administered intravenously. After general endotracheal anesthesia, the abdominal skin was sterilized and draped. Pneumoperitoneum was achieved with a Veress needle inserted at Palmer's point, which lies $3 \mathrm{~cm}$ below the left costal margin in the midclavicular line and represents the point at which intraabdominal adhesions are least likely [5]. A $10 \mathrm{~mm}$ port was then placed at this point followed by an additional $5 \mathrm{~mm}$ port placed under direct vision in the left side of the abdomen. A $30^{\circ}$ laparoscope was placed through the $10 \mathrm{~mm}$ port, laparoscopic examination was performed, and any abnormalities were noted.

In the absence of contraindications, the incarcerated contents were reduced through a combination of blunt and sharp dissection with scissors. No attempt was made to remove the hernia sac. A $2 \mathrm{~mm}$ incision was placed within the umbilical 
Table 1. All Reports of Laparoscopic Hernia Repair Using Two-Port Technique (2005 - 2017)

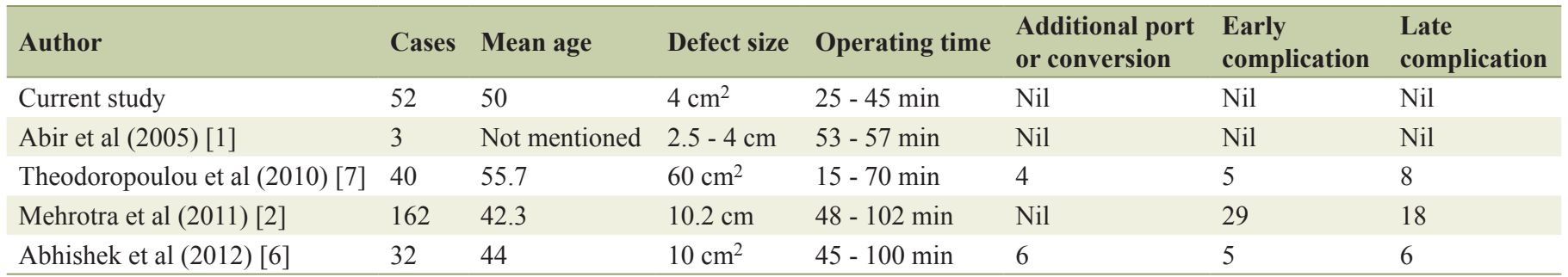

skin fold and a suture passing instrument was used to close the umbilical defect. Using the device, and under direct vision, Ethicon suture was introduced into the abdominal cavity on one side of the defect and retrieved back on the other side of the defect. At least two sutures were placed across the fascial defect, and the sutures were then tied and the suture knots buried under the skin.

An appropriately sized Parietex mesh was chosen to adequately close the defect with an overlap of $2 \mathrm{~cm}$ circumferentially. Four 2-0 polydiaxonesulphate (PDS) sutures were placed through the polypropylene side of the mesh at the corners. The mesh was then rolled and inserted through the $10 \mathrm{~mm}$ port into the abdominal cavity, unrolled inside the abdomen, and positioned with the polypropylene side against the abdominal wall and the polytetrafluoroethylene side down towards the abdominal contents. Pneumoperitoneum was decreased to $10 \mathrm{mmHg}$ and, with a suture-passing instrument, the corresponding pairs of sutures were individually pulled transabdominally through appropriately placed $2 \mathrm{~mm}$ skin incisions. The sutures were pulled tight and the mesh was raised to the abdominal wall. A $2 \mathrm{~cm}$ overlap was once again confirmed, and the anchoring sutures were tied in the subcutaneous tissues. The mesh edges were fixed using a helical tacker at $3 \mathrm{~cm}$ intervals. Pneumoperitoneum was released and ports sites were closed.

\section{Results}

The study population consisted of 20 males and 32 females, none of whom had had any previous abdominal surgery. Their mean age was 50 years (30 - 65 years). All patients had a body mass index (BMI) below $30 \mathrm{~kg} / \mathrm{m}^{2}$. All patients underwent hernia repair via the two-port technique. None required additional ports or conversion to the conventional method. The average defect size was $4 \mathrm{~cm}^{2}$ (range $2-10 \mathrm{~cm}^{2}$ ). The mean operating time was $32 \mathrm{~min}$ (range 25 - $45 \mathrm{~min}$ ). There was no iatrogenic injury, significant procedural blood loss, or subcutaneous emphysema at either port site. Patients were allowed enteral feeding as early as $3 \mathrm{~h}$ following surgery. All patients were routinely discharged on the second postoperative day. Upon direct questioning on discharge, all the patients reported being happy due to rapid and comfortable recovery. Moreover, patients were satisfied with the esthetic outcome due to barely visible scars. Patients were scheduled follow-up on the seventh day and 3 months following surgery; all patients attended for day 7 follow-up and reported no complications and, out of 45 of 52 patients attending their 3-month follow-up appointment, none reported any complications.

\section{Discussion}

Many surgeons have now successfully practised two-port laparoscopic umbilical hernia repair with safe and superior results to three- or four-port hernia repair in terms of pain, cosmesis, and patient acceptance $[1,2]$. Various techniques and special instruments including suture-passing instruments (e.g., CarterThomason and Gore devices) have been used to accomplish the procedure without the need for additional ports [1,2]. Twoport laparoscopic umbilical hernia repair has several methodological variations, but all have the same main common steps as described herein. Our approach does not significantly differ from other two-port approaches but the study highlights how proper patient selection and experience and proficiency in laparoscopic hernia repair help to achieve optimal results.

Although the technique used here was safe, it does have some limitations. For example, the two-port technique should not be used in cases in which technical difficulties are anticipated or encountered such as in patients with dense peritoneal adhesions or trapped/strangulated umbilical hernias. Therefore, a feasibility assessment is advisable before attempting the two-port technique, and difficult cases should routinely revert to the three- or four-port method or even the conventional technique. Likewise, if there is intraoperative bleeding, there should be a low threshold to convert to the standard three-port technique. Meticulous dissection and gentle instrument handling are sine qua non for safe and successful completion of the procedure.

A number of studies have examined two-port minimally invasive umbilical hernia repair techniques, and these are summarized in Table $1[1,2,6,7]$. Overall, our results were comparable with previous studies in terms of operating time and small defect sizes. Abir et al [1] reported the first laparoscopic hernia repair using a two-port technique in three cases in 2005. Mehrotra et al [2] reported the largest series involving 162 cases, in which technical success was $100 \%$ with no conversions to the open or the three-port procedure. Abhishek et al [6] examined a series of 32 patients treated with two access ports but, in contrast to our technique, mesh fixation was accomplished with a transabdominal suture alone to reduce procedural costs. Some comparative studies have reported favorable cost analyses of laparoscopic versus open umbilical hernia repair, with laparoscopic umbilical hernia repair significantly 
reducing costs compared to open mesh repair [8], albeit with the type of mesh used and fixation device having a considerable impact on cost calculations. The preferred mesh fixation method used during laparoscopic umbilical hernia remains controversial; some surgeons prefer to use tacks in order to reduce the surgical time and postoperative pain [9], while others prefer to use a transabdominal suture alone in order to reduce the costs and to reduce recurrence rates due to the higher tensile holding strengths of sutures compared to tacks [10].

Chronic pain after laparoscopic umbilical hernia repair is common, disabling, and can be challenging to treat. Some authors argue that the use of tacks significantly reduces postoperative pain, with pain generally reported to be worse after repair with sutures than with tacks [6]. Sutures penetrate the full thickness of the abdominal wall musculature and fascia, which is thought in some cases to cause local muscle ischemia and consequently severe postoperative pain [11]. We used transabdominal sutures to close the umbilical defect and fix the mesh together with tacks to reduce or to avoid postoperative pain, and none of our patients reported chronic pain. The laparoscopic approach is reported to have a recurrence rate of $10 \%$ [12-14] due to inadequate mesh fixation, infection, lateral detachment of the mesh, inadequate overlap, and inadequate mesh [15]. However, with careful case selection and precise technique and patience, the two-port laparoscopic umbilical hernia repair technique can become an amazing reality in one's own surgical practice. This is the second largest reported series of two-port umbilical hernia repair and the first reported German study on the use of the two-port technique in laparoscopic umbilical hernia repair.

In conclusion, careful patient selection is important in order to obtain optimal outcomes from two-port laparoscopic umbilical hernia repair. Experience and proficiency in performing laparoscopic hernia repair are also important factors contributing to achieving excellent results without complications.

\section{Acknowledgments}

We would like to thank all the patients who participated in this study.

\section{Conflicts of Interest}

The authors declare that they have no competing interests.

\section{Author Contributions}

GA and AO operated on the patient, conception and design, data acquisition, data analysis and interpretation, drafting the article, critical revision of the article, and final approval of the version to be published.

\section{Financial Disclosures}

None.

\section{References}

1. Abir F, Eisenberg D, Bell R. Laparoscopic ventral hernia repair using a two $(5-\mathrm{mm})$ port technique. JSLS. 2005;9(1):94-96.

2. Mehrotra PK, Ramachandran CS, Arora V. Two port laparoscopic ventral hernia mesh repair: an innovative technical advancement. Int J Surg. 2011;9(1):79-82.

3. Liberman MA, Rosenthal RJ, Phillips EH. Laparoscopic ventral and incisional hernia repair: a simplified method of mesh placement. J Am Coll Surg. 2002;194(1):9395.

4. Heniford BT, Park A, Ramshaw BJ, Voeller G. Laparoscopic ventral and incisional hernia repair in 407 patients. J Am Coll Surg. 2000;190(6):645-650.

5. Palmer R. Safety in laparoscopy. J Reprod Med. 1974;13(1):1-5.

6. Abhishek V, Mallikarjuna MN, Shivaswamy BS. Laparoscopic umbilical hernia repair: technique paper. ISRN Minimally Invasive Surg. 2012:906405.

7. Theodoropoulou K, Lethaby D, Hill J, Gupta S, Bradpiece H. Laparoscopic hernia repair: a two-port technique. JSLS. 2010;14(1):103-105.

8. Beldi G, Ipaktchi R, Wagner M, Gloor B, Candinas D. Laparoscopic ventral hernia repair is safe and cost effective. Surg Endosc. 2006;20(1):92-95.

9. Wassenaar EB, Raymakers JT, Rakic S. Impact of the mesh fixation technique on operation time in laparoscopic repair of ventral hernias. Hernia. 2008;12(1):23-25.

10. Eid GM, Prince JM, Mattar SG, Hamad G, Ikrammudin $\mathrm{S}$, Schauer PR. Medium-term follow-up confirms the safety and durability of laparoscopic ventral hernia repair with PTFE. Surgery. 2003;134(4):599-603.

11. Gillian GK, Geis WP, Grover G. Laparoscopic incisional and ventral hernia repair (LIVH): an evolving outpatient technique. JSLS. 2002;6(4):315-322.

12. Heniford BT, Ramshaw BJ. Laparoscopic ventral hernia repair: a report of 100 consecutive cases. Surg Endosc. 2000;14(5):419-423.

13. Toy FK, Bailey RW, Carey S, Chappuis CW, Gagner M, Josephs LG, Mangiante EC, et al. Prospective, multicenter study of laparoscopic ventral hernioplasty. Preliminary results. Surg Endosc. 1998;12(7):955-959.

14. Koehler RH, Voeller G. Recurrences in laparoscopic incisional hernia repairs: a personal series and review of the literature. JSLS. 1999;3(4):293-304.

15. Awad ZT, Puri V, LeBlanc K, Stoppa R, Fitzgibbons RJ Jr, Iqbal A, Filipi CJ. Mechanisms of ventral hernia recurrence after mesh repair and a new proposed classification. J Am Coll Surg. 2005;201(1):132-140. 